\title{
Fast Transient Buck Converter Using a Hysteresis PWM Controller
}

\author{
Yong-Xiao Liu*, Jin-Bin Zhao ${ }^{\dagger}$, and Ke-Qing Qu ${ }^{*}$ \\ ${ }^{*}$ College of Electrical Engineering, Shanghai University of Electric Power, Shanghai, China
}

\begin{abstract}
In this paper, a fast transient buck converter using hysteresis PWM control is presented. The proposed control method is based on hysteresis control of the capacitor $\mathrm{C}$ voltage. This offers a faster transient response to meet the challenges of the power supply requirements for fast dynamic input and load changes. It also provides better stability and solves the compensation problem of the error amplifier in conversional voltage PWM control. Finally, the steady-state and dynamic operation of the proposed control method are analyzed and verified by simulation and experimental results.
\end{abstract}

Key words: Fast transient response, Feedback, Feed-forward, Hysteresis control

\section{INTRODUCTION}

Nowadays, dc-dc converters play an important role in industry, the air-space industry, portable systems, distributed generation, electrical devices, electrical machines, power factor correction and voltage regulation [1], [2], [10]-[12]. Thus, high-quality power supplying converters that can accomplish low-cost and high-efficiency performance are in demand [3]. High accuracy, fast transient response and enhancing driving capability are expected when a converter operates in the pulse width modulation (PWM) mode [4], [5]. Buck converters, as one of the widely used converters, have been investigated with different structures and control methods [3]-[16]. The widely used PWM control takes several switching cycles, due to its duty-cycle limitation, to recover the output voltage in response to step changes in the loading current [6]. For the popular PWM control buck converter operating in continuous conduction mode (CCM), a complicated compensation network is needed to ensure stable operation due to the existence of complex poles in the loop gain transfer function [7]. Due to changes in the duty cycle and load condition, a change in the open loop transfer function will bring many difficulties for designing the optimum values of the parameters in the phase compensator.

Manuscript received Feb. 7, 2013; revised Jul. 26, 2013

Recommended for publication by Associate Editor Yong Kang.

Corresponding Author: zhaojinbin@shiep.edu.cn

Tel: +86-21-35305346, Shanghai University of Electric Power

*College of Electrical Engineering, Shanghai University of Electric Power, China
To avoid these difficulties in design, current mode control is applied together with voltage feedback to improve the stability and dynamic performance [9]. In this case, many electric components including a current sensing resistor are needed. Several approaches have been reported in the literature. Hysteresis dc-dc converters have a fast transient response since the hysteresis $\mathrm{dc}-\mathrm{dc}$ converter does not require an error amplifier or external compensation components, which may slow down the transient response. Thus, the system size and chip die area are smaller than those of conventional PWM voltage-mode and current-mode dc-dc converters because of the removal of the external compensation components [3], [8].

An improved hysteresis PWM control method using a triangle waveform modulated by output voltage feedback and input voltage feed-forward was proposed. The proposed control method only uses a comparator with hysteresis, a feedback resistor, and a feed-forward resistor. Thus, the number of components in the control circuit are obviously reduced. In addition, since an error amplifier is not used, the stability of the converter is improved. When the input voltage is changed, it has a smaller peak value and a faster regulation speed than the conventional control method [5],[10].

The operations of the control method are described in Section 2 , the steady-state and dynamic characteristics of the proposed converter are analyzed in Section 3, and the fast transient response and good regulation were verified by the experiment and PSIM simulation in Section 4. 


\section{OPERATING PRINCIPLES}

The configuration of a buck converter with a synchronous rectifier employing the proposed control scheme is shown in Fig.1. The control circuit consists of a comparator $U$ with hysteresis and a feedback resistor $R_{f}$. Based on the hysteresis characteristic curve of comparator $U$, the key waveforms of comparator $U$ in one switching cycle are shown in Fig.2. The output voltage is returned to capacitor $C$ for the triangular wave generator through resistor $R_{f}$. The feed-forward voltage $V_{I}$ is connected to capacitor $C$ through the feed-forward resistor $R$. The feed-forward voltage $V_{l}$ is also returned to comparator $U$ with hysteresis through resistor $R_{2}$. The control circuit operates as follows. When the output voltage or input voltage becomes large, the charging current of capacitor $C$ in the on mode period increases and the discharging current of capacitor $C$ in the off mode period decreases. Thus, the on time duration of the pulse decreases, and the off time duration increases. In addition, when the input voltage $V i$ becomes large, the threshold voltage levels $V_{H}$ of comparator $U$ become large. Thus, the change of the input voltage can directly affect the threshold voltage $V_{H}$ to rapidly regulate the duty cycle, not through the output voltage change caused by the input voltage. Therefore, the switching duty was changed, and the output voltage can be regulated. It is worth noting that the feed-forward voltage $V_{l}$ has two key roles in the control circuit. On the one hand it can quickly reflect the changes of the input voltage with a $(1-D) T S$ time delay under the worst condition. Therefore, it has the feed-forward control effect. $D$ is the duty ratio and $T_{s}$ is the cycle time. On the other hand, it has two kinds of values based on the switch state, that is the input voltage $V i$ and low level voltage. Thus, the threshold levels of comparator $U$ were generated and vary with voltage $V_{l}$. As a result, it can achieve better response results than the conventional PWM control scheme.

\section{OPERATION ANALYSIS}

\section{A. Steady-state Analysis}

To simplify the analysis, all of the circuit elements are assumed to be ideal. In Fig. $1, V_{f}$ is the voltage across capacitor $C, V_{L}$ and $V_{H}$ are the threshold levels of comparator $U$, and $V_{U}$ is the output voltage of comparator $U$. The switching cycle starts at instant $\mathrm{t}=0$.

(i) state 1: $0<t<T_{O N}$

When the output signal $V_{U}$ is high level, the following equations are obtained:

$$
i=C \frac{d V_{f}}{d t}=\frac{k V_{i}-V_{f}}{R}+\frac{V_{o}-V_{f}}{R_{f}}
$$

By solving the above equations under the initial condition of $V_{f}(0)=V_{L}$, the next equation is obtained:

$$
V_{f}=V_{L} e^{\frac{-1}{R_{p} C} t}+\frac{k \times V_{i} R_{p}}{R}\left(1-e^{\frac{-1}{R_{p} C} t}\right)+\frac{V_{o} R_{p}}{R_{f}}\left(1-e^{\frac{-1}{R_{p} C} t}\right)
$$

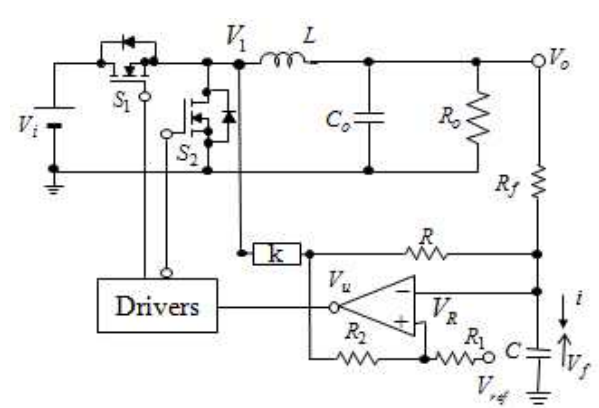

Fig. 1. Circuit diagram of the proposed converter.

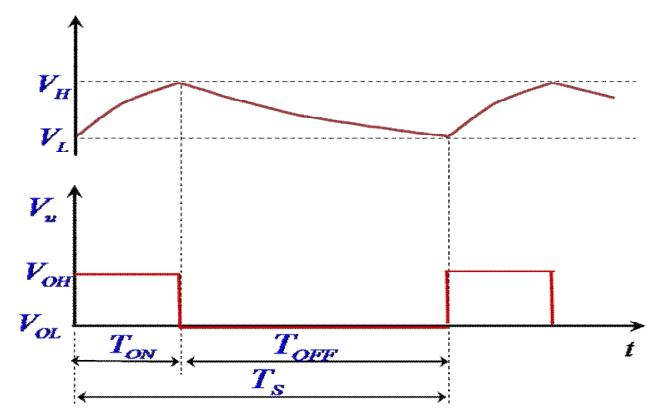

Fig. 2 .Key waveforms of the comparator.

$$
R_{p}=\frac{R R_{f}}{R+R_{f}}
$$

From equation (2), $V_{f}$ increase exponentially from $V_{L}$ to $R_{p} k V_{i} / R+R_{p} V_{o} / R_{f}$. Since $V_{f}$ must be greater than $V_{H}$ to invert the state of the comparator, the next constraint is obtained:

$$
\frac{R_{p}}{R} k V_{i}+\frac{R_{p}}{R_{f}} V_{o}>V_{H}
$$

By setting $V_{f}=V_{H}$ and $t=T_{O N}$ in equation (2), $T_{O N}$ is obtained:

$$
T_{O N}=C R_{p} \ln \frac{R_{p} k V_{i} / R+R_{p} V_{o} / R_{f}-V_{L}}{R_{p} k V_{i} / R+R_{p} V_{o} / R_{f}-V_{H}}
$$

If $V_{H^{-}} V_{L}<<R_{p} k V_{i} / R+R_{p} V_{o} / R_{f}-V_{H}$, equation (4) can be approximated as follows:

(ii ) state 2: $T_{O N}<t<T_{s}$

$$
T_{O N} \simeq C R_{p} \frac{V_{H}-V_{L}}{R_{p} k V_{i} / R+R_{p} V_{o} / R_{f}-V_{H}}
$$

When output signal $V_{U}$ is low level, the following equations are obtained:

$$
i=C \frac{d V_{f}}{d t}=\frac{-V_{f}}{R}+\frac{V_{o}-V_{f}}{R_{f}}
$$

By solving the above equations under the initial condition of $V_{f}\left(T_{O N}\right)=V_{H}$, the next equation is obtained:

$$
V_{f}=\frac{V_{o} R_{p}}{R_{f}}+\left(V_{H}-\frac{V_{o} R_{p}}{R_{f}}\right) e^{\frac{-1}{R_{p} C}\left(t-T_{O N}\right)}
$$

From equation (7), $V_{f}$ decreases exponentially from $\mathrm{V}_{\mathrm{H}}$ to $R_{p} V_{o} / R_{f}$. Since $V_{f}$ must be lower than $V_{L}$ to invert the state of the comparator, the next constraint is obtained: 


$$
\frac{R_{p}}{R_{f}} V_{o}<V_{L}
$$

From the equation (7), $T_{O F F}$ is obtained by setting $V_{f}=V_{L}$ and $t=T_{S}$ :

$$
T_{\text {OFF }}=C R_{p} \ln \frac{V_{H}-R_{p} V_{o} / R_{f}}{V_{L}-R_{p} V_{o} / R_{f}}
$$

If $V_{H^{-}} V_{L}<<V_{L}-R_{p} V_{o} / R_{f}$, equation(9) can be approximated as follows:

$$
T_{\text {OFF }} \simeq C R_{p} \frac{V_{H}-V_{L}}{V_{L}-R_{p} V_{o} / R_{f}}
$$

By combining (4) and (9), the duty ratio $D=T_{O N} /\left(T_{O N}+T_{O F F}\right)$ is:

$$
D=\frac{\ln \frac{V_{c}+k k_{1} V_{i}-V_{L}\left(k_{1}+1\right)}{V_{c}+k k_{1} V_{i}-V_{H}\left(k_{1}+1\right)}}{\ln \left(\frac{V_{c}+k k_{1} V_{i}-V_{L}\left(k_{1}+1\right)}{V_{c}+k k_{1} V_{i}-V_{H}\left(k_{1}+1\right)} \times \frac{V_{c}-V_{H}\left(k_{1}+1\right)}{V_{c}-V_{L}\left(k_{1}+1\right)}\right)}
$$

where, $k_{1}=R_{f} / R$.

And cycle $T s$ is :

$$
T_{S}=C R_{p} \ln \left(\frac{\left(\frac{R_{p}}{R} k V_{i}+\frac{R_{p}}{R_{f}} V_{c}-V_{L}\right)\left(V_{H}-\frac{R_{p}}{R_{f}} V_{c}\right)}{\left(\frac{R_{p}}{R} k V_{i}+\frac{R_{p}}{R_{f}} V_{c}-V_{H}\right)\left(V_{L}-\frac{R_{p}}{R_{f}} V_{c}\right)}\right)
$$

By combining (5), (10) and $D=T_{O N} /\left(T_{O N}+T_{O F F}\right)$ the output voltage is obtained as follows:

$$
\begin{aligned}
V_{o} & =\frac{R_{f}\left(R_{1}+R_{2}\right)-R_{1}\left(R+R_{f}\right)}{R_{2} R_{f}-R R_{1}} V_{i} d^{*} \\
\text { Where, } d^{*} & =\frac{R_{2} V_{r e f} /\left(R_{1}+R_{2}\right)-R V_{o} /\left(R+R_{f}\right)}{k V_{i}\left(R_{f} /\left(R+R_{f}\right)-R_{1} /\left(R_{1}+R_{2}\right)\right)} \\
V_{L} & =R_{2} V_{r e f} /\left(R_{1}+R_{2}\right) \\
V_{H} & =R_{2} V_{\text {ref }} /\left(R_{1}+R_{2}\right)+R_{1} k V_{i} /\left(R_{1}+R_{2}\right)
\end{aligned}
$$

In addition, the output voltage can be written as:

$$
V_{o}=\frac{V_{i} V_{L}}{V_{i} R_{p} / R_{f}+V_{L}-V_{H}+k V_{i} R_{p} / R}
$$

\section{B. Dynamic Analysis}

To further analyze the dynamic characteristics of the buck converter circuit, the small signal circuit model is obtained by applying PWM switching modeling techniques. Fig. 3 shows the small-signal equivalent circuit of a buck converter with the proposed control scheme. In Fig.3, $K_{l}$ is the gain of the feed-forward circuit, $K_{2}$ is the gain of the feedback circuit, $r_{s}$ is the $O N$ resistance of the switch, and $r_{c}$ is the series equivalent resistance of capacitor $C$. Based on Fig.3, the small-signal transfer functions can be obtained. Each open-loop transfer function is defined and derived as follows.

The input to output transfer functions are expressed as:

$$
G_{v}=\frac{\Delta v_{o}}{\Delta v_{i}}=\frac{D\left(1+S C r_{c}\right)}{S^{2} L C+S\left[\left(r_{c}+r_{s}\right) C+1 / R\right]+1}
$$

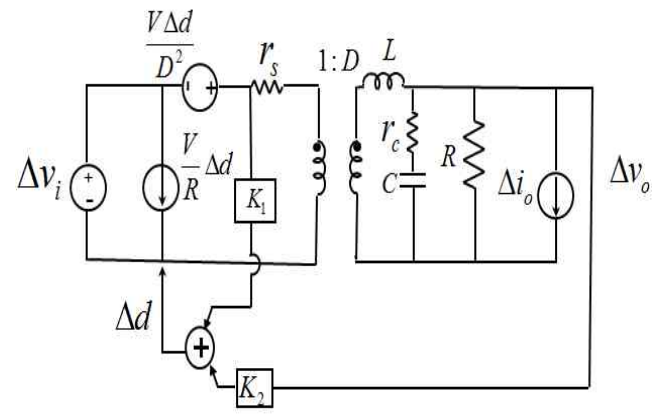

Fig. 3. Small signal equivalent circuit.

The duty cycle to the output voltage transfer function is expressed as:

$$
G_{d}=\frac{\Delta v_{o}}{\Delta d}=\frac{v_{i}\left(1+S C r_{c}\right)}{S^{2} L C+S\left[\left(r_{c}+r_{s}\right) C+1 / R\right]+1}
$$

It is well known that the output impedance of the converter has a resonance peak due to the LC resonance of the filter circuit. This resonance causes large undershoot and overshoot voltages on the output during load changes. The output impedance transfer function is expressed from the small signal analysis as:

$$
Z_{o}=\frac{\Delta v_{o}}{\Delta i_{o}}=\frac{S L\left(1+S C r_{c}\right)}{S^{2} L C+S\left[\left(r_{c}+r_{s}\right) C+1 / R\right]+1}
$$

Where, $\Delta v_{o}, \Delta v_{i}, \Delta d$ and $\Delta i$ are small perturbations in the output voltage $v_{o}$, the input voltage $v_{i}$, the switch duty ratio $d$ and the output current $i_{o}$.

In addition, by combining equation (5), equation (10), and $K_{l}=\Delta d / \Delta v i, K_{2}=\Delta d / \Delta v_{o}$, the next equation can be written as:

$$
\begin{gathered}
K_{1}=\frac{R V_{o} /\left(R+R_{f}\right)-R_{2} V_{r e f} /\left(R_{1}+R_{2}\right)}{k V_{i}^{2}\left(\frac{R_{f}}{R+R_{f}}-\frac{R_{1}}{R_{1}+R_{2}}\right)} \\
K_{2}=\frac{-R /\left(R+R_{f}\right)}{k V_{i}\left(\frac{R_{f}}{\left(R+R_{f}\right)}-\frac{R_{1}}{\left(R_{1}+R_{2}\right)}\right)}
\end{gathered}
$$

The audio susceptibility $G(s)$ is then expressed as follows:

$$
G=\frac{G_{v}-K_{1} G_{d}}{1+T}
$$

Where, $T=K_{2} G_{d}$.

$$
T=\frac{940 * 60 * 10^{-9} s+60}{9.4 * 10^{-9} s^{2}+(1 / 3+0.1504) * 10^{-4} s+1}
$$

Fig. 4 shows a control block diagram of the circuit proposed in this paper. As can be seen from the block diagram, the feed-forward pass from the input to the duty control eliminates the effect of input voltage variations. Hence, the output voltage is not influenced by fluctuations of the input voltage.

Fig. 5 shows the results of the frequency responses of the open loop feedback control transfer function taking the proportion coefficient $k$ as a parameter. Fig. 6 shows the feed-forward control transfer function taking the proportion coefficient $k$ as a parameter. The frequency responses of the 


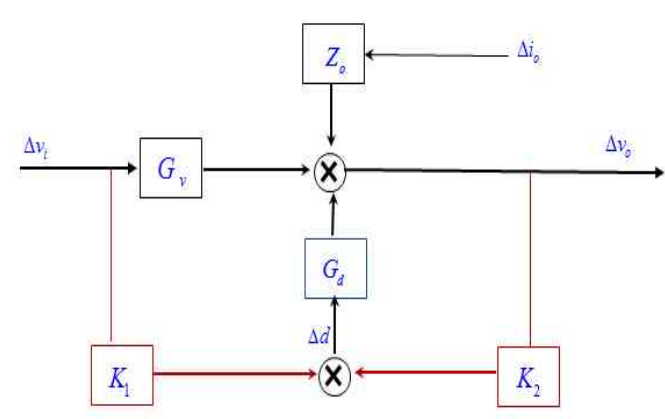

Fig. 4. Control block diagram of the buck converter employing the proposed control scheme.

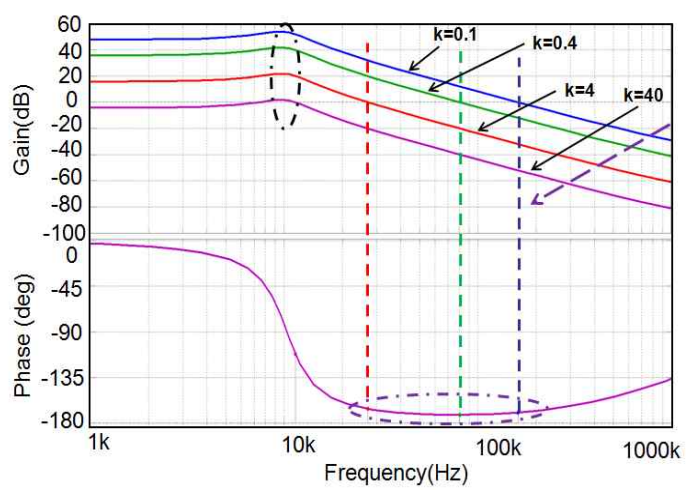

Fig. 5. Frequency responses of the open loop feedback control taking $k$ as a parameter.

gain and phase show that the control loop is steady and has a good phase margin with the $k$ increases. The frequency responses of the gain and phase show that the gain, in both the low and high frequencies, decreases with the increase in the coefficient constant $k$. The phase characteristics are the same here because no differences are observed among any of the values of $k$.

Fig. 7 shows a comparison of the results of the frequency responses of the audio susceptibility $G(s)$. It can be seen in this figure that the gain of the audio susceptibility $G(s)$ is well decreased by employing the proposed feed-forward control method. The feed-forward control has an advantage especially when the change of an external disturbance is rapid because its information is transferred to the controller without delay. On the other hand, the feed-forward path is an open loop and the control result is not corrected by this method. Therefore, it is important to design the parameters (like the proportion coefficient $k$ ) of the feed-forward path properly in order to obtain an excellent control characteristic. The value of $K_{l}$ in the audio susceptibility $G(s)$ without feed-forward control is zero. As a result, the feed-forward control has a lower loop gain. This can be shown by equation (20). Here, the phase characteristics are the same.

Fig.8 shows the frequency responses of $G(s)$ taking $k$ as a parameter. The resonance peak of $G(s)$ is well increased with an increase in $k$ while the resonant frequency is reduced. All of

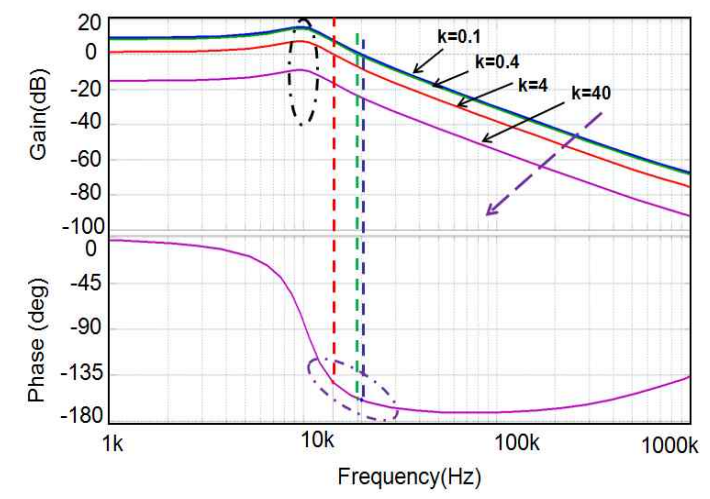

Fig. 6. Frequency responses of the open loop feed-forward control taking $k$ as a parameter.

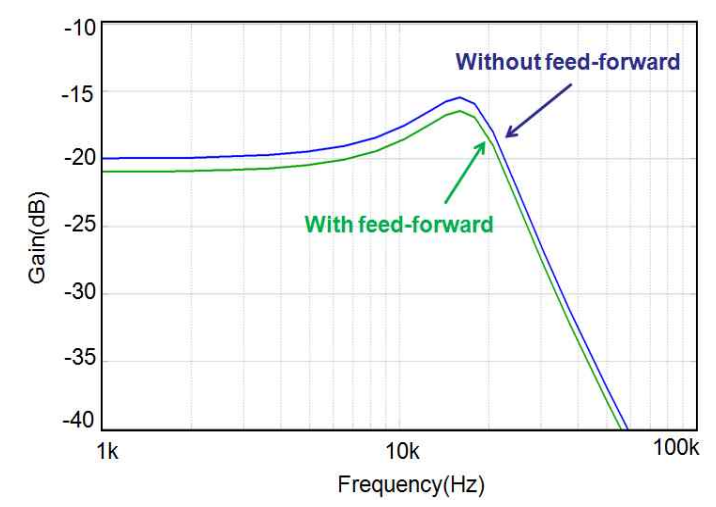

Fig. 7. Frequency responses of $G(s)$.

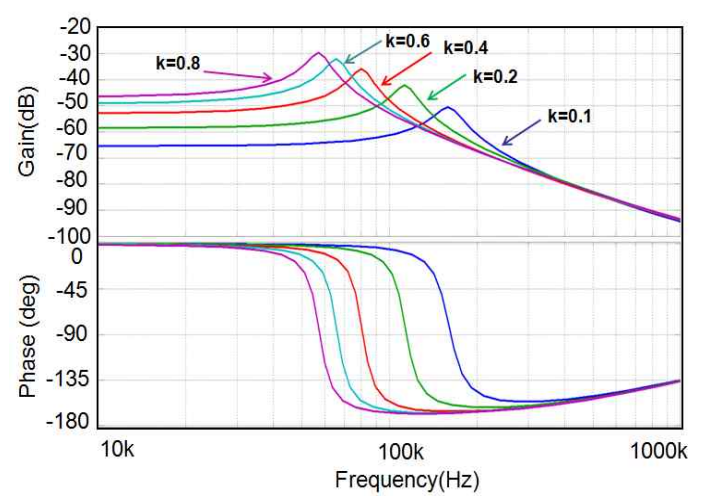

Fig. 8. Frequency characteristics of $G(s)$ for $k$.

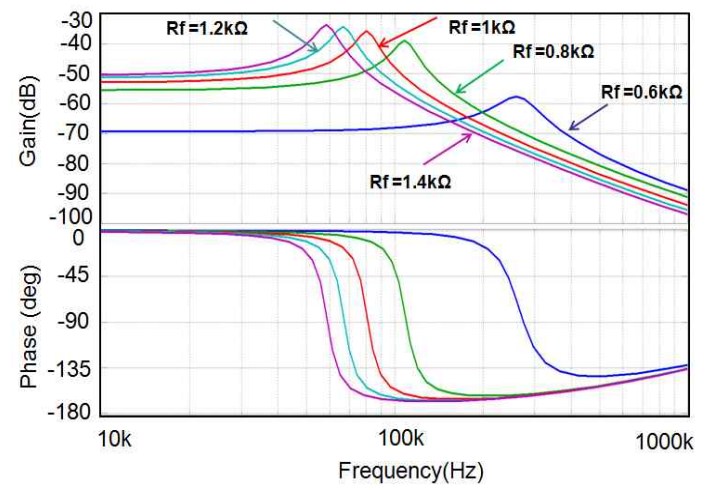

Fig. 9. Frequency characteristics of $G(s)$ for $R_{f}$. 


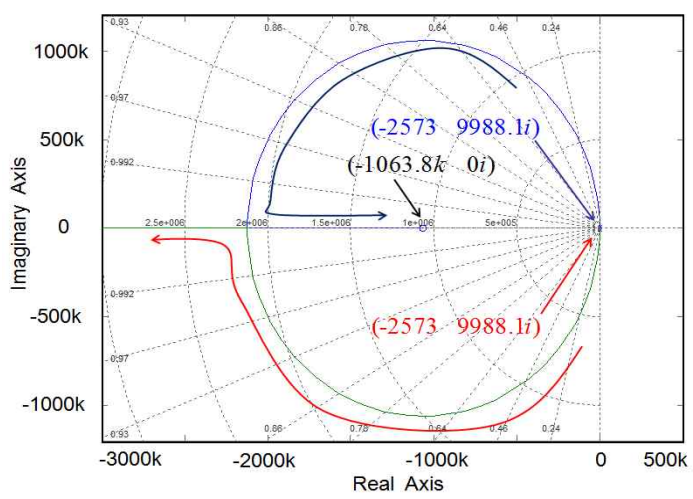

Fig. 10. Root locus of the open loop transfer function.

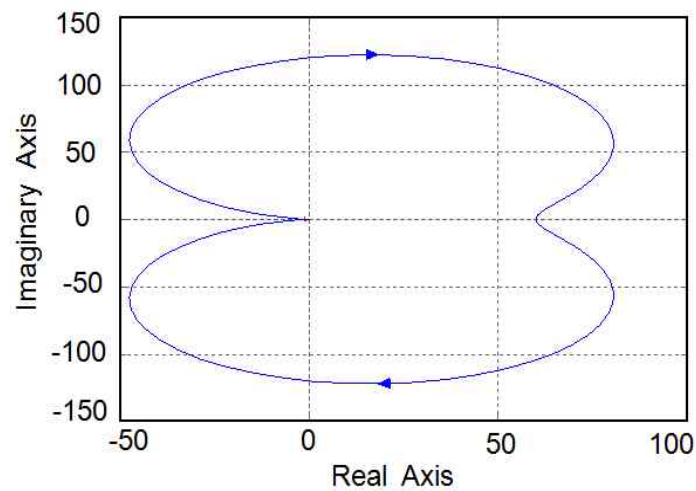

(a)

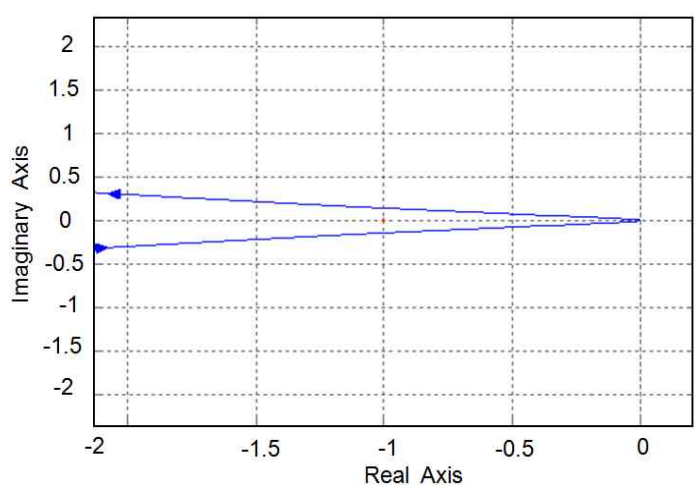

(b)

Fig. 11. (a) Nyquist diagram of the transfer function. (b) The amplification region at the point $(-1,0 i)$.

them have good gain characteristics by employing the proposed feed-forward control method.

For the value of $R_{f}$, it is not easy to determine the proper value analytically. The frequency responses of $G(s)$, taking $R_{f}$ as a parameter, are shown in Fig.9. It is obvious that the resistance $R_{f}$ should be smaller for increasing the resonant frequency. It is a trade-off taking the value of $R_{f}$ or $k$ between the gain of $G(s)$ and the resonant frequency.

Note that the above calculations were carried out by introducing the internal resistance and ESR of the output capacitor.
According to the open loop transfer function (Equation 21), the open loop root locus can be shown as Fig.10. It can be seen that the curvature of the root locus is a circle. The open loop transfer function has two conjugate complex poles and one zero. Actually, the two poles are far from the imaginary axis and strong damping is obtained. The separation point is farther from the imaginary axis and the root locus bends to the left. Thus, fast attenuation oscillation processes and fast steady features are achieved. In addition, a small overshoot can be achieved. In this paper the pole point is $1000 *(-2.5730+$ $9.9881 i)$ and $1000 *(-2.5730-9.9881 i)$.

Fig. 11 (a) shows a Nyquist diagram of the transfer function. It can be seen that point $(-1,0 i)$ is not surrounded by the Nyquist plot. In addition, there are no pole points of the open transfer function in the right half plane. The stability of the buck converter control system is obtained in terms of the Nyquist stability criterion. Fig.11( b) shows the part of the amplification region at point $(-1,0 i)$ of the Nyquist plot. It can be seen that point $(-1,0 i)$ is not surrounded or passed through by the Nyquist plot.

If the duty cycle can be obtained, the output voltage can also be obtained by equation $v_{o}=v_{i}^{*} d$. According to equation (11), the parameters have been given except for the value of $k$. The accurate value of $k$ can be calculated after solving a nonlinear equation by the Numerical algorithm. Here, the calculated exact value range of $k$ is $0.471<k<0.475$, and the maximum error of the output voltage was $\pm 0.175 \%$.

To keep the system in the steady state, the value of $k$ is very important. Here, the range of $k$ can be calculated through equation (20). The characteristic root of the system characteristic equation $(1+T(s))$ cannot but has strict negative real parts. Based on that, the range of $k$ can be $\mathrm{k}>0$ according to the Routh criterion.

There are two loops in the control system. The feed-forward control realizes the correction of the input voltage changes and the direct regulation of the duty cycle. Especially, the feedback control obtains rapid regulation of the duty cycle when the load is changed. The input to the output equation can be written as:

$$
G=\frac{G_{v}-K_{1} G_{d}}{1+T} V_{i}+\frac{G_{d}}{1+T} D
$$

Where, $T=K_{2} G_{d}$.

To achieve a result where the output voltage variation has a minor effect on the output voltage, feed-forward correction with full compensation can be used in the control system. The equation can be written as:

$$
\frac{G_{v}-K_{1} G_{d}}{1+T} V_{i}=0
$$

Here, if $G_{v}-K_{l} G_{d}=0$, feed-forward correction with full compensation can be achieved. Finally, the value range of $k$ should be $0.4951<k<0.4967$, and the maximum error was \pm $0.3 \%$. 


\section{SIMULATION AND EXPERIMENTAL RESULTS}

To verify the proposed control method, a simulation model based on Fig. 1 has been built in PSIM and a prototype has been designed. The circuit parameters are shown in Table I. The conventional PWM controller is a TL5001 PWM controller. The regulator makes use of the PI compensation network.

Fig. 12(a) shows the relation between the output current and the output voltage. As can be seen in Fig. 12(a), the variations of the output voltage are very small. The simulation values of the output voltage are in good agreement with the experimental values.

Fig. 12(b) shows the relation between the input voltage and the output voltage. It can be seen that the variation of the output voltage is very small even when the input voltage varies significantly.

It is observed that no steady-state error appears in the output voltage when there are changes of the input voltage and load current even when the controller does not employ a high-gain operational amplifier.

Fig. 13(a) and Fig. 13(b) show the responses of the proposed controller and conventional controller undergoing $8 \mathrm{~V}$ to $5 \mathrm{~V}$ and $5 \mathrm{~V}$ to $8 \mathrm{~V}$ input voltage step changes. To see the output clearly, the conventional control output voltage $\left(V_{o} 1\right)$ is made by adding $0.2 \mathrm{~V}$. It can be seen that the proposed controller has a smaller peak value and a faster transient recovery time than the conventional controller.

Fig. 14(a) and Fig. 14(b) show the simulated and experimental responses of the proposed controller and conventional controller undergoing $5 \mathrm{~A}$ to $2 \mathrm{~A}$ and $2 \mathrm{~A}$ to $5 \mathrm{~A}$ load current step changes, respectively. It can be seen that the steady-state error becomes very small and that the employment of the proposed controller reduces the peak value of the output voltage.

Fig. 15(a) and Fig. 15(b) give the performance of the hysteresis band change when the input voltage changes. It shows that the feed-forward control has an effect on the hysteresis band to achieve a fast dynamic response. The proposed control and the constant hysteresis band control are shown in red and blue, respectively. It can be seen from these results that the transient voltages for the variation of the input voltage are improved by introducing the proposed control.

Fig. 16(a) and Fig. 16(b) show the gain and phase frequency characteristics of the control to the output transfer function, respectively. From the bode plots of the feed-forward control to the output and the feedback control to the output transfer function, the dynamic characteristic is approved well.

\section{CONCLUSIONS}

A new hysteresis control method with voltage feed-forward and feedback has been proposed and analyzed in this paper. The proposed control method is based on the hysteresis control

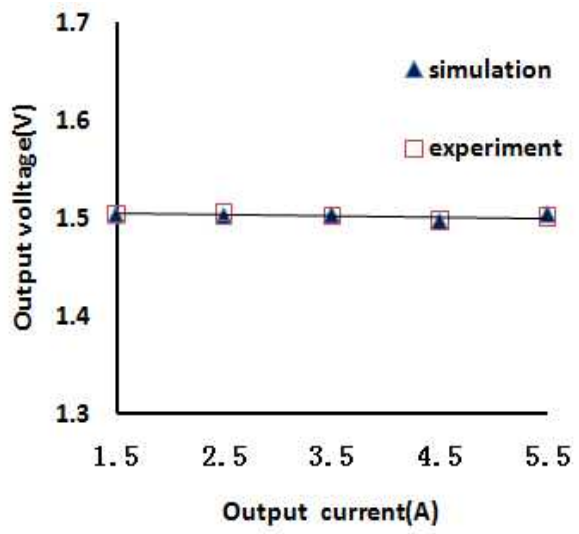

(a)

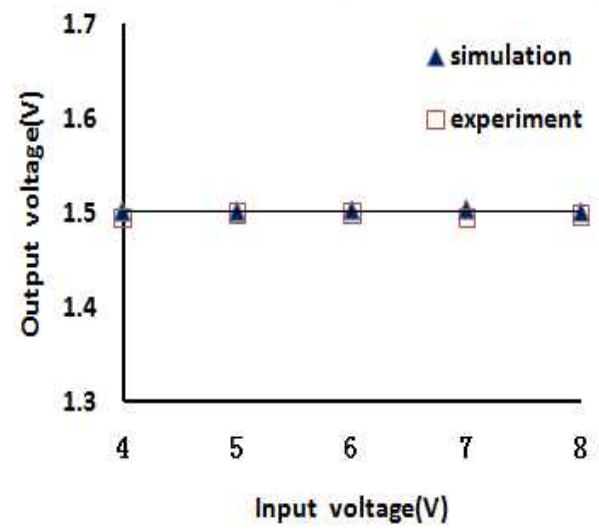

(b)

Fig. 12. (a) Relation of the output current and output voltage (b) Relation of the input voltage and output voltage

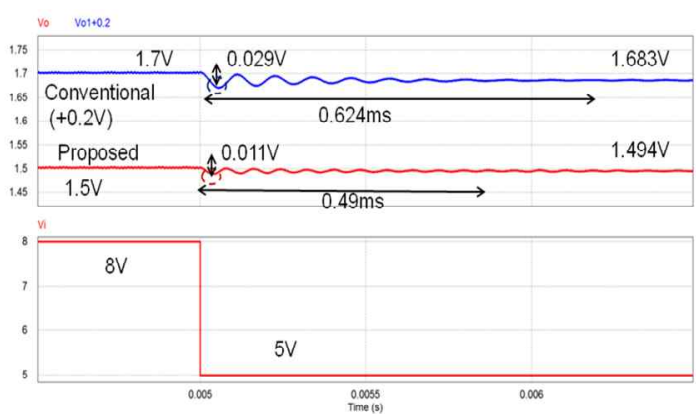

(a)

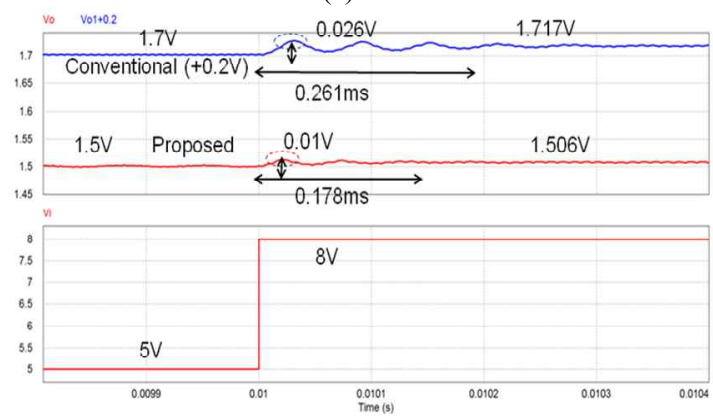

(b)

Fig. 13. (a) Simulated response to a $8 \mathrm{~V}$ to $5 \mathrm{~V}$ input voltage step change. (b) Simulated response to a $5 \mathrm{~V}$ to $8 \mathrm{~V}$ input voltage step change. 


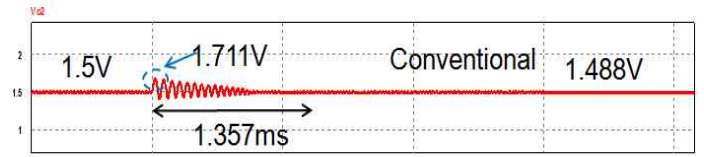

\begin{tabular}{|c|c|c|}
\hline $1.5 \mathrm{~V} \leftarrow 1.627 \mathrm{~V}$ & Proposed & $1.495 \mathrm{~V}$ \\
\hline$\stackrel{\underset{0739 \mathrm{~ms}}{\longrightarrow}}{\stackrel{4}{\longrightarrow}}$ & & \\
\hline
\end{tabular}
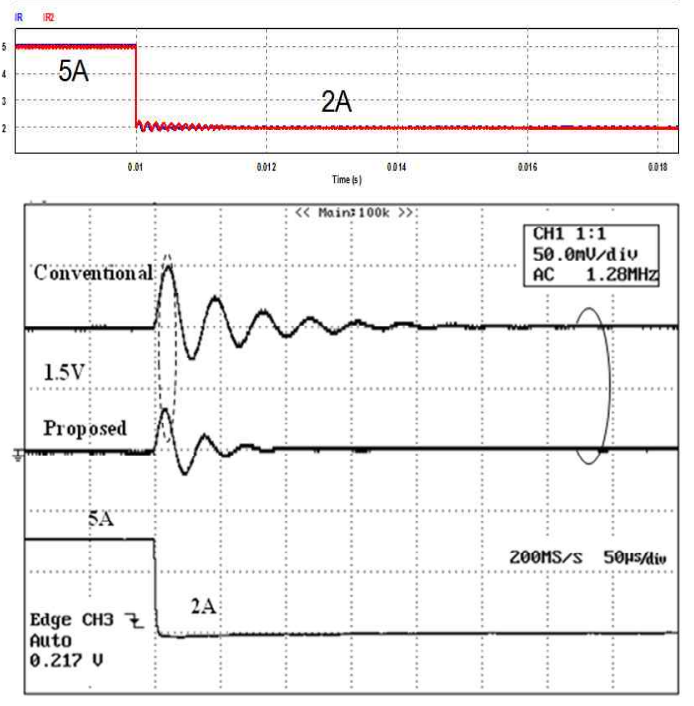

(a)
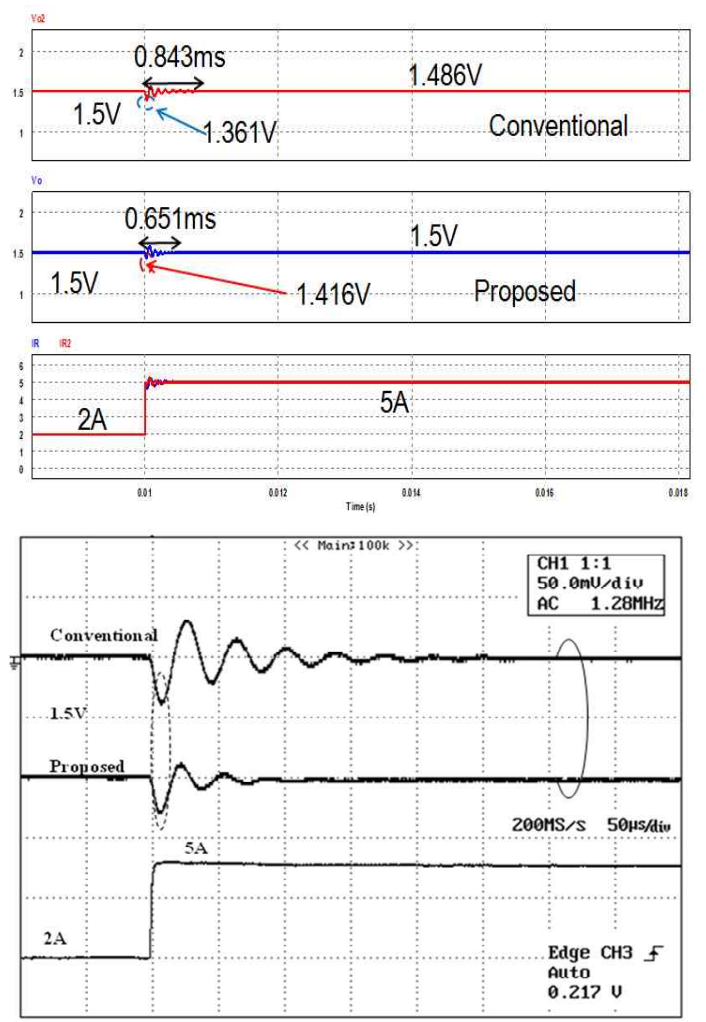

(b)

Fig. 14. (a) Simulated and experimental response to a 5 Ato $2 \mathrm{~A}$ load current step change. (b) Simulated and experimental response to a $2 \mathrm{~A}$ to $5 \mathrm{~A}$ load current step change.

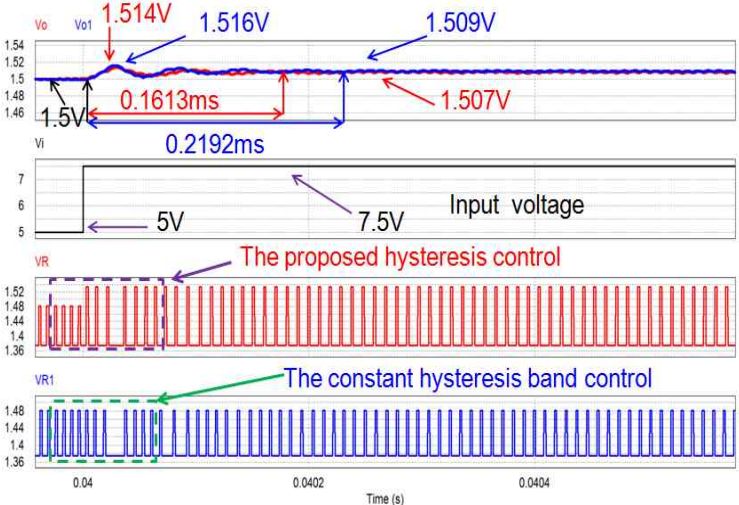

(a)

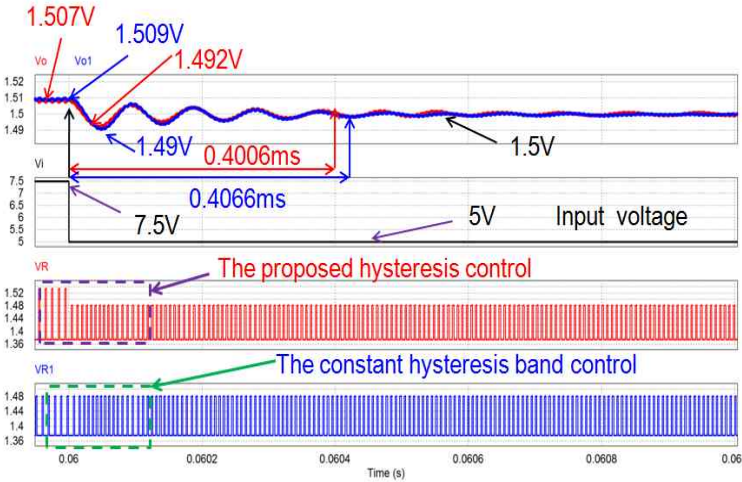

(b)

Fig. 15 . (a) $5 \mathrm{~V}$ to $7.5 \mathrm{~V}$ input voltage step change. (b) $7.5 \mathrm{~V}$ to $5 \mathrm{~V}$ input voltage step change.

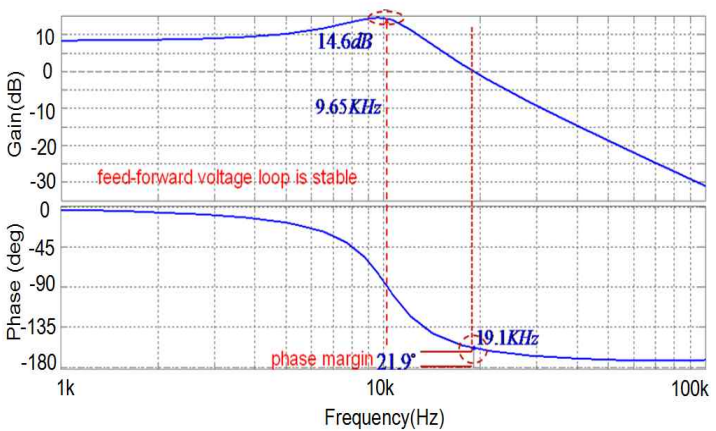

(a)

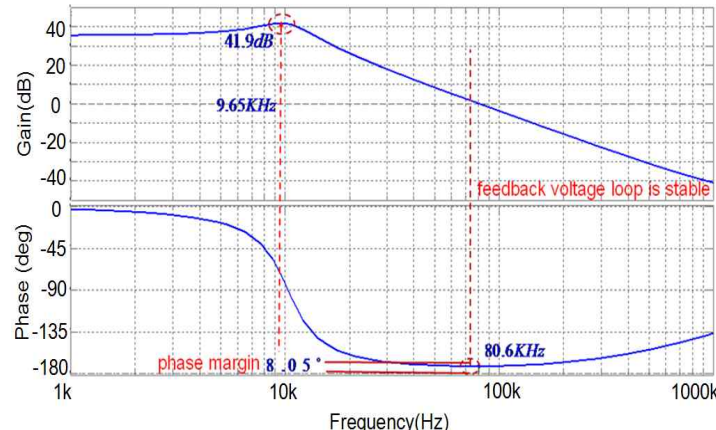

(b)

Fig.16 (a) Bode plots of feed-forward control to output transfer function.(b) Bode plots of feedback control to output transfer function 
Table I

Design Parameters of THe Proposed Converter

\begin{tabular}{|cc|}
\hline Parameters & Values \\
DC Source Voltage $V_{i}$ & $5 \mathrm{~V}$ \\
Output Voltage $V_{o}$ & $1.5 \mathrm{~V}$ \\
Resistance $R_{l}$ & $560 \Omega$ \\
Resistance $R_{2}$ & $10 \mathrm{k} \Omega$ \\
Filter Inductance $L$ & $20 \mathrm{uH}$ \\
Comparator Resistance $R$ & $10 \mathrm{k} \Omega$ \\
Capacitance $C$ & $2200 \mathrm{pF}$ \\
Filter Capacitance $C_{o}$ & $470 \mathrm{uF}$ \\
Reference Voltage $V_{r e f}$ & $1.455 \mathrm{~V}$ \\
Feedback Resistance $R_{f}$ & $1 \mathrm{k} \Omega$ \\
Proportion Coefficient $k$ & 0.4 \\
\hline
\end{tabular}

of the capacitor $C$ voltage. It offers a faster transient response to meet the challenges of the power supply requirements for fast dynamic input and load changes. In addition, it provides better stability and solves the compensation problem of the error amplifier in the conversional voltage PWM control. The proposed control method only uses a comparator with hysteresis, a feedback resistor and a feed-forward resistor. Therefore, the number of components in the control circuit are obviously reduced. In addition, since an error amplifier is not used, the stability of the converter is better. When the input voltage is changed, it has a smaller peak value and a faster regulation speed than the conventional control method. The proposed control method achieves a good regulation and transient response in the buck converter for input and load transients. The dynamic characteristics of the buck converter circuit and the small signal circuit model are obtained by applying PWM switching modeling techniques. The steady-state and dynamic operation of the proposed control method are analyzed and verified by simulation and experimental results. The transient performance of the output voltage for large variations in the input voltage is improved by introducing feed-forward control by using proper values for $k$ in the feed-forward path. The gain of the audio susceptibility $G(s)$ is significantly decreased by employing the proposed feed-forward control method.

In order to investigate the dynamic performances of the proposed PWM controller, a small signal analysis was carried out by introducing the averaging technique. The frequency responses of the gain and phase show that the open loop control is steady and has a good phase margin when $k$ increases. The frequency responses of the gain and phase show that the gains in both the low and high frequency decrease with the increase of the coefficient constant $k$. The resonance peak of $G(s)$ is well reduced with an increase of $k$, while the resonant frequency is also reduced. All of these obtain a good gain by employing the proposed feed-forward control method. The resistance $R_{f}$ should be smaller for increasing the resonant frequency. There is a trade-off when taking the value of $R_{f}$ or $k$ between the gain of $G(s)$ and the resonant frequency.

It is worth mentioning that the feed-forward voltage $V_{l}$ has two key roles in the control circuit. On the one hand, it can quickly reflect the changes of the input voltage with a $(1-D) T_{s}$ time delay under the worst condition. As a result, has the feed-forward control effect. $D$ is the duty ratio and $T_{s}$ is the cycle time. On the other hand, it has two kinds of values based on the switch state, that is the input voltage $V_{i}$ and the low level voltage. Thus, the threshold level of comparator $U$ was generated and varies with voltage $V_{l}$. The proposed method can achieve better response results than the conventional PWM control scheme.

\section{ACKNOWLEDGMENT}

The authors would like to acknowledge the financial support of the Program for Professor of Special Appointment (Eastern Scholar) at Shanghai Institutions of Higher Learning and Shanghai talent development fund (Grant No2012024). and Leading Academic Discipline Project of Shanghai Municipal Education Commission (J51303).

\section{REFERENCES}

[1] I.-D. Kim, J.-Y. Kim, E.-C. Nho, and H.-G. Kim, "Analysis and design of a soft-switched pwm sepic dc-dc converter," Journal of Power Electronics, Vol. 10, No.5, pp.461-467,Sep.2010.

[2] W. R. Liou, M. L. Yeh, and Y. L. Kuo, "A high efficiency dual-mode buck converter integrated circuit for portable applications," IEEE Trans. Power Electron, Vol. 23, No.2, pp. 667-677, Mar. 2008.

[3] H. H. Huang, C. L. Chen, and K. H. Chen, "Adaptive window control (AWC) technique for hysteresis DC-DC buck converters with improved light and heavy load performance," IEEE Trans. Power Electron, Vol. 24, No. 6, pp. 1607-1617, Jun. 2009.

[4] K. H. Chen, H. W. Huang, and S. Y. Kuo, "Fast transient DC-DC converter with on-chip compensated error amplifier," IEEE Trans. Circuits Syst. II, Vol. 54, No. 12, pp. 1150-1154, Dec. 2007.

[5] M. Siu, P. K. T. Mok, K. N. Leung, Y. H. Lam, and W.-H. $\mathrm{Ki}$, "A voltage-mode PWM buck regulator with end-point prediction," IEEE Trans.on Circuits Syst. II, Vol. 53, No. 4, pp. 294-298, Apr. 2006.

[6] Y. Q Zheng, H. Chen, and K. N. Leung, "A fast-response pseudo-PWM buck converter with PLL-based hysteresis control," IEEE Trans. Very Large Scale Integr. (VLSI) Syst.,Vol. 20, No. 7, pp. 1167-1174, Jul. 2012.

[7] X. H. Wu and X. B. Wu, "Adaptive hysteresis window control (AHWC) technique for hysteresis DC-DC buck converter with constant switching frequency," Power and Energy Engineering Conference, 2010 Asia-Pacific, pp. $1-4,2010$

[8] M. Castilla, L. Garcia de Vicuna, J. M. Guerrero, J. Miret, and N. Berbel, "Simple low-cost hysteresis controller for 
single-phase synchronous buck converters," IEEE Trans. Power Electron, Vol. 22, No. 4, pp. 1232-1241, Jul. 2007.

[9] T. Nabeshima, T. Sato, K. Nishijima, and S. Yoshida, "A novel control method of boost and buck-boost converters with a hysteresis PWM controller," in Proc.EPE, pp. 1-6, 2005.

[10] A. Khaligh, A. M. Rahimi, and A. Emadi, "Modified pulse-adjustment technique to control $\mathrm{dc} / \mathrm{dc}$ converters driving variable constant-power loads," IEEE Trans. Ind. Electron, Vol. 55, No. 3, pp. 1133-1146, Mar. 2008.

[11] H. Y. Cha, R. J. Ding, Q. S. Tang, and F. Z. Peng, "Design and development of high power dc-dc converter for metro vehicle system," IEEE Trans. Ind. Appl., Vol. 44, No. 6, pp. 1795-1804, Dec. 2008.

[12] M. G. Villava and E. F. Ruppert, "Input-controlled buck converter for photovoltaic applications: modeling and design," in Proc. PEMD, pp.505-509, 2008.

[13] R. Mohsen, L. W. Zhou, and M. Nasser, "Improvement of one-cycle controller response with a current mode controller," Journal of Power Electronics, Vol. 10, No. 1, pp.461-467, Jan. 2010.

[14] S. C. Huerta, P. Alou, J. A. Oliver, O. García, J.A. Cobos, and A. M. Abou-Alfotouh, "Nonlinear control for DC-DC converters based on hysteresis of the $c_{\text {out }}$ current with a frequency loop to operate at constant frequency," IEEE Trans. Ind. Electron, Vol. 58, No. 3, pp. 1036-1043, Mar. 2011.

[15] S. C. Huerta, P. Alou, O. Garcia, J. A. Oliver, R. Prieto, and J. Cobos, "Hysteresis mixed-signal controller for high-frequency DC-DC converters operating at constant switching frequency," IEEE Trans. Power Electron, Vol. 27, No. 6, pp. 2690-2696, Jun. 2012

[16] S. Kapat and P.T. Krein, "Improved time optimal control of a buck converter based on capacitor current," IEEE Trans. Power Electron, Vol. 27, No. 3, pp. 1444-1454, Mar. 2012.

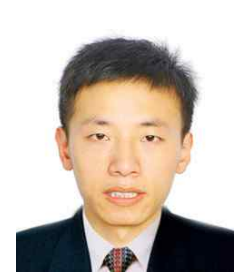

Yong-Xiao Liu was born in China, in 1988. $\mathrm{He}$ will receive his M.S. in Electrical Engineering from the Shanghai University of Electric Power, Shanghai, China, in 2014. $\mathrm{He}$ is a Student Member of the China Power Supply Society. His current research interests include power electronics and control.

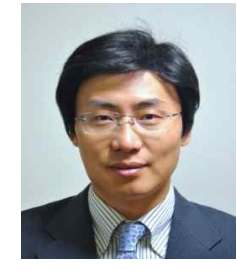

Jin-Bin Zhao (M'06) was born in China, in 1972. He received his M.S. and Ph.D. in Electrical Engineering from Oita University, Oita, Japan, in 2002 and 2005, respectively. He worked as a Researcher at the R\&D Headquarters of the Origin Electric Co., Ltd, Japan, from 2005 to 2011 . He is currently a Professor at the Shanghai University of Electric Power, Shanghai, China. His current research interests include the control of power converters, soft-switching power converters, inverters, distributed power systems, power-factor correction and electric drive systems. He currently holds three U.S. patents, five Japanese patents and five Chinese patents. He has published 40 technical papers in journals and conference proceedings. Dr. Zhao is a Member of the Institute of Electrical and Electronics Engineers (IEEE) and the Institute of Electrical Engineers of Japan (IEEJ), the Institute of Electronics Information and Communication Engineers of Japan (IEICE) and a Senior Member of the China Power Supply Society.

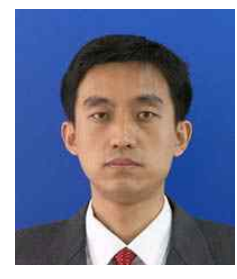

Ke-Qing Qu was born in China, in 1970. He received his Ph.D. in Electrical Engineering from Shanghai University, Shanghai, China, in 2004. He is currently an Associate Professor at the Shanghai University of Electric Power, Shanghai, China. $\mathrm{He}$ is also a Master Instructor. As a visiting scholar, he studied in Germany with full funding by the national foundation for study, from 2009 to 2010. His current research interests include power electronic conversion, and new energy generation and its application to power systems. He holds one Chinese patent, one monograph and has more than 30 academic theses, 12 of which were included in the international Engineering Index. 